\title{
MICROESTRUCTURA DISCURSIVA Y SEGMENTACIÓN INFORMATIVA EN LA CONVERSACIÓN COLOQUIAL
}

\author{
(a Pablo, el benjamín)
}

\author{
Antonio Hidalgo Navarro \\ Universitat de València. Grupo Val.Es.Co. \\ Antonio.Hidalgo@uv.es
}

\section{Resumen}

The aim of this article is to describe the minimum units of conversational structure. These minimum units, the Act and the Subact, take part in the segmentation of conversation in information units, that allow to recognize a hierarchical organization in our linguistic emissions.

There are some segmental and suprasegmental marks that delimit the extent of Acts and, in the other hand, there are also several pragmatic functions that allow to see dicerent kinds of Subacts.

In summary, Act and Subact are the first step to analyze the oral discourse and to set up the socalled Conversational Grammar.

\section{La estructura de la conversación y su organización jerárquica}

Uno de los aspectos fundamentales asumibles en el estudio de toda lengua, ya sea en su forma oral, ya sea en su forma escrita, es el análisis de sus textos y discursos posibles. Es necesario partir de ciertos patrones de medición, disponer de un "sistema métrico" que nos capacite para establecer las segmentaciones oportunas en cada caso. Este es y ha sido, tradicionalmente, el cometido de la Gramática, y más precisamente, de la Sintaxis, que ha contado con unidades de medida idóneas para la detección de parcelas lingüísticas adecuadas. Se habla así del sintagma, la oración, el texto, etc., si bien su determinación ha estado siempre vinculada al ámbito del lenguaje escrito, es decir a la consideración de la lengua como producto estático y acabado.

No se trata ahora de determinar el grado de adecuación de tales unidades a sus respectivos ámbitos de acción, si bien es cierto que habitualmente han funcionado con cierto nivel de precisión. No obstante, el problema inmediato de este "sistema métrico oracional" viene a manifestarse al desplazar el enfoque desde el sistema a la actuación. Recuérdense en este sentido las observaciones de ciertos analistas en orden a rechazar la unidad oración en el ámbito de uso, y su preferencia por otras entidades, como p.e. el enunciado (Gutiérrez, 1984).

1 El presente trabajo ha contado para su realización con la subvención derivada del Proyecto de investigación BFF2000-1438 "Diccionario de Particulas del Español" auspiciado por el Ministerio de Ciencia y Tecnología. 
En este estado de cosas, conocido es el desarrollo que han experimentado los estudios de Pragmática a lo largo de las dos últimas décadas, de modo que se ha intensificado e incluso privilegiado el estudio de la actuación, del ámbito del uso, o lo que es lo mismo, del lenguaje oral, de la lengua como proceso dinámico en marcha. Así, en el seno de estas nuevas líneas de estudio se ha planteado la misma necesidad de análisis, si bien aquí el reconocimiento de parcelas ha afectado, no al texto escrito, sino a lo que genéricamente se ha denominado Discurso Oral.

No es este el lugar adecuado para extenderse con relación al proceso de reconocimiento de estas unidades de análisis, por lo que en la exposición que sigue nos vamos a ceñir a un tipo de discurso oral específico, particularmente interesante por su naturaleza misma, la conversación, más exactamente, la conversación coloquial. La cuestión inicial sería determinar, pues, tras el planteamiento previo, qué sistema métrico podría ser el más apropiado para la delimitación de las unidades propias de la conversación.

En este sentido, el Grupo Val.Es.Co. (véase nota 2) ha planteado un modelo demarcativo que contempla de forma notoria las diversas maneras en que el componente fónico suprasegmental puede llegar a articular el flujo de habla, unas veces de modo esencial, otras veces subsidiariamente, estableciendo cierta jerarquía entre sus diferentes unidades ${ }^{3}$ (para una descripción global de dicho sistema de segmentación resulta de obligada consulta el trabajo de Briz y otros, 2003). De este modo, el presente trabajo abordará fundamentalmente la des-

2 Para el Grupo Val.Es.Co. (Valencia, Español Coloquial) de la Universitat de València la conversación es un tipo de discurso que se caracteriza por los siguientes rasgos: es oral, es decir, se articula a través del canal fónico; dialogal, lo que implica, frente al monólogo, sucesión de intercambios; inmediato, puesto que, a diferencia de un informativo o un mensaje pregrabado, se desarrolla en la coordenada espacio-temporal aqui-ahora-ante ti; retroalimentado y cooperativo, puesto que se obra juntamente con otro y su intervención; dinámico, como demuestra la alternancia de turnos, que además es no predeterminada, a diferencia de otros discursos dialogales tales como el debate, la entrevista, etc. La conversación es coloquial cuando presenta además los rasgos no planificado, lo que implica un escaso control de la producción de habla, que favorece la presencia de reinicios, vacilaciones y vueltas atrás; no transaccional, es decir, orientada a un fin interpersonal, de comunión fática, frente a la conversación transaccional, constituida como medio para obtener un fin específico. Consecuentemente, el tono de dicha conversación es informal. En suma, en la llamada conversación coloquial se reconocen, por un lado, rasgos conversacionales, relativos al tipo de discurso y, por el otro, rasgos coloquiales, propios del registro de uso.

Los rasgos coloquiales, a su vez, vienen favorecidos y están determinados por la situación comunicativa, en concreto por parámetros tales como la relación de igualdad social y funcional entre los interlocutores, su relación vivencial de proximidad (conocimiento mutuo compartido), un marco de interacción familiar y la temática no especializada de la interacción. La mayor o menor presencia de tales parámetros o rasgos situacionales determina grados de coloquialidad. De otro modo, lo coloquial se constituye en una escala gradual: a mayor presencia de todos estos rasgos, mayor coloquialidad (menor planificación, mayor carácter interpersonal y mayor informalidad). Ello significa que no todas las conversaciones presentan el mismo grado de coloquialidad; unas, con mayor presencia de los rasgos anteriores, se acercarán al prototipo de lo coloquial; otras, con menor presencia, se alcjarán del prototipo, constituyendo la periferia de lo coloquial. De ahí la distinción que proponíamos en Briz (coord.) (1995) entre conversaciones coloquiales prototipicas y conversaciones coloquiales periféricas. Estas últimas, sin dejar de ser coloquiales, se sitúan en un punto de la escala más o menos alejado del prototipo, es decir, en la periferia.

3 El grupo Val.Es.Co. establece dos tipos de unidades en la conversación coloquial: unidades de orden social y unidades de orden estructural. Las primeras dependen de la estructura social o externa de la conversación, esto es, de la existencia de dos o más hablantes que intercambian sus papeles conversacionales en el proceso de interacción. Por su parte, las unidades de orden estructural tienen un carácter interno y son inherentes a la conversación misma: vienen dadas por el desarrollo de la conversación y no por factores sociales o externos. Sobre estas cuestiones véanse las extensas explicaciones de Briz (2000a), Briz (2000b), Briz (en prensa), Pons (2001) o Briz y Grupo Val.Es.Co. (en prensa). 
cripción de las unidades mínimas de análisis, y el establecimiento de bases y criterios para su reconocimiento. En todo caso, y aunque dadas las limitaciones de espacio no haremos aquí una descripción global de todas las unidades conversacionales, sí convendría esbozar al menos las líneas maestras de nuestro sistema de segmentación.

Se distinguen dos niveles estructurales, en primer término el Dialógico, cuyas unidades son el Diálogo (como unidad máxima), el Intercambio (como sucesión de intervenciones de diversos hablantes) y la Intervención, unidad dialogal mínima. En segundo término se halla el nivel Monológico, cuya unidad máxima es la Intervención, esto es, emisión que se puede constituir como inicio de habla posterior, reacción a una intervención precedente o reacción e inicio a la vez ${ }^{4}$. No vamos a entrar, por la necesaria limitación de espacio en otras disquisiciones sobre la constitución de la Intervención y su identificación por parte del grupo Val.Es.Co; a lo sumo podemos evidenciar que, al margen del cambio de voz, otros recursos que pueden ayudar a identificar la Intervención vienen dados por la presencia de ciertas marcas lingüísticas como la entonación (p.e. una inflexión descendente marcada puede contribuir decisivamente a reconocer el final de una Intervención), la posesión de fuerza ilocutiva, etc.

Por lo demás, si nos adentramos más en este Nivel Monológico, en el interior de una intervención es posible reconocer uno o varios segmentos específicos; véase así (1):

(1)

A: Cállate

B: No, porque no me da la gana. Estoy harto de tu prepotencia

donde podemos diferenciar, en primer término, una unidad monologal menor que la Intervención, el Acto (constituyente inmediato de aquella) capaz de funcionar aisladamente en un contexto dado y de constituirse como intervención por si mismo. Efectivamente, la intervención (1A) cumple estos requisitos, mientras que la intervención (1B) agrupa dos segmentos aislables en iguales condiciones que (1A), a saber, "No, porque no me da la gana" y "Estoy harto de tu prepotencia".

Obsérvese, sin embargo, que, dada la imposibilidad de aislar el fragmento "porque no me da la gana" en ese contexto (no podría constituirse por sí solo como reacción lingüística de $\mathrm{B}$ a A), debemos considerar dicho segmento como parte integrante de un Acto. A pesar de ello percibimos que tal fragmento aporta información pertinente (en este caso de índole justificativa) para la viabilidad del Acto en sí (efectivamente, el sentido del Acto cambiará sensiblemente si prescindimos de dicho segmento); es evidente además que el fragmento

4 La identificación de una intervención en la conversación implica la identificación de un "cambio de voz", en cuyo caso podremos considerar una intervención reactiva; este criterio no es, sin embargo, suficiente para llegar a delimitar intervenciones iniciativas. En este sentido cabria entender una intervención prototípica como aquella que se constituye a la vez como reacción a una intervención precedente e inicio desencadenante de posteriores intervenciones. Permancce, sin embargo, el problema de la delimitación de intervenciones iniciativas, ya que en ocasiones no es obligado el cambio de voz para que se produzca un cambio de intervención; p.e. cuando el hablante decide voluntariamente cambiar el tema de la conversación, con lo que de algún modo está rompiendo la continuidad de su intervención y abriendo una nueva intervención, aunque, obviamente, sin cambio de voz. De cualquier forma, hay que pensar que un cambio de tópico, de estrategia, implica la existencia de dos intervenciones. La intervención es, pues, una estrategia; para cumplir ese fin se pueden seguir, incluso, caminos diferentes que conducen, finalmente, a la misma meta (véase al respecto Briz y Grupo Val.Es.Co. en prensa, y Briz y otros, 2003). 
en cuestión se halla delimitado por un tonema demarcativo coincidente con el final de una estructura sintáctica y entonativa completa (descenso melódico pronunciado, sentido aseverativo categórico). Pues bien, este tipo de fragmentos reciben en nuestra propuesta la denominación de Subactos, es decir, subunidades integrantes del Acto, reconocibles como aportes o soportes informativos relevantes, pero no aislables en el contexto dado.

\section{Definición y reconocimiento de Actos}

El Acto se define, pues, como unidad estructural monologal, jerárquicamente inferior a la Intervención. Todo Acto tiene, comunicativamente hablando, la capacidad de ser aislable en un contexto dado, esto es, puede constituirse por sí mismo en Intervención y funcionar aisladamente en ese contexto; posee, pues, capacidad de aislabilidad. El Acto, además, es identificable y reconocible entre el conjunto de habla circundante; queda definido así como la menor unidad de habla capaz de funcionar aislada en el contexto discursivo real en que se produce.

El Acto posee por lo demás valor modal completo, es decir, una única función ilocutiva específica (pregunta, rechazo, etc.) y unidad melódica, ya que su curva entonativa resulta completa en sí misma (la aseveración p.e. presenta un tonema descendente de carácter final), lo que, por otro lado, no significa que todo elemento con contorno melódico propio constituya necesariamente un Acto. En cuanto al reconocimiento de una unidad como Acto, existe una serie de marcas, tanto lingüísticas (gramaticales o léxicas) como prosódicas, que contribuyen a ello y que pueden incluso coaparecer, si bien suele percibirse cierta preeminencia de unas sobre otras 5 .

\section{1. Índices lingüísticos segmentales demarcativos de Actos}

Cualquier palabra puede llegar a funcionar como Acto siempre que cumpla los requisitos previamente aludidos. En este sentido, existen elementos que parecen especialmente habilitados para desempeñar esta función, como ocurre con algunas proformas o adverbios tales como eso, así, no, sí, etc., que funcionan de modo aislado con cierta asiduidad y, por tanto, suelen constituirse como Actos ${ }^{6}$ :

12 E6: \#yo tengo unos principios\# y \#para mí hay valores fundamentales\#§

$13 \mathrm{G} 3$ :

$\S$ \#eso\#/ \#y tú los sigues ¿'no?\#

Sin embargo, esto no siempre es así, como en el caso de las formas si o no, cuando funcionan como fórmulas discursivas de iniciación del turno; en tal circunstancia no deben ser consideradas como Actos independientes, ya que carecen de capacidad de aislabilidad. Así ocurre en $42 \mathrm{G} 17$ de (3):

5 Para más detalles sobre las caracteristicas específicas de estas marcas de balizamiento véase Briz y otros (2003).

6 Los ejemplos que irán apareciendo a continuación proceden del corpus de conversaciones coloquiales de Val.Es.Co. y siguen, por tanto, sus convenciones de transcripción. El símbolo \# representa los límites inicial y final de un Acto. 
(3)

40 E21: \#lo sé porque no tengo ni puta idea\#/ \#porque éel\# $\$$

$41 \mathrm{~L} 10$ :

$\S$ \#ies raro?\#

42 G17: \#no/ oye/ que desde pequeño pues le han daoo\#// \#más o menos desde pequeño $\downarrow$ le han obligao $\downarrow \#$ \# al final / pues se ha acostumbrao\#/ yy

Resulta evidente que el empleo de no en este contexto no supone realmente una negación a la pregunta inmediatamente previa, sino más bien una forma de introducir atenuadamente la intervención, aportando a la vez cierto matiz de concesividad ("aunque <él> pueda parecer raro no lo es").

Otras veces es la presencia de un verbo explicito el principal factor que nos permite reconocer un Acto, ya que dicho verbo pone de manifiesto la fuerza ilocutiva de tal Acto:

te prometo que iré al cine contigo ${ }^{7}$

Por su especial comportamiento discursivo, los denominados conectores pragmáticos se constituyen con asiduidad como marcas lingüísticas fiables para la delimitación de Actos, si bien no todos, ni tampoco en todos los casos. En definitiva, el tipo de unidad que introduzca el conector (o marcador en sentido más amplio) suele depender del tipo de constituyente discursivo en que se enmarque su aptitud funcional: puede enlazar, pues, Actos o Subactos ${ }^{8}$. Ahora bien, si enlaza Subactos su función ya no será la de conector, sino la de conjunción. Será conveniente, pues, plantear una división clara entre las nociones de conjunción y de marcador (o conector): las conjunciones, a diferencia de los conectores, sólo enlazan Subactos; no pueden enlazar Actos.

Asimismo, es necesario establecer algunas diferencias funcionales entre los marcadores, según su papel demarcativo específico. Hay así conectores que pueden constituirse como Actos en sí mismos o bien comportarse como marcas demarcativas de Actos (según el contexto y su capacidad o incapacidad para ser aislables en dicho contexto), y existen otros conectores que solo pueden funcionar como marcas delimitativas de Actos (o de Subactos), pero sin llegar nunca a constituirse como Actos en sí mismos.

Al primer grupo corresponden p.e. ciertos modalizadores, como bueno, capaz de funcionar por sí solo como Acto si cumple los requisitos exigibles (p.e. bueno puede constituirse simplemente como un Acto afirmativo). Si no posee dicha capacidad de aislabilidad no debemos considerarlo como Acto específico, p.e. en estructuras con valor concesivo similares a (5B):

\footnotetext{
7 Obsérvese en este sentido la opinión de Briz (en prensa): "un proverbo como decir, que se usa con múltiples valores ilocutivos, puede servirnos de prueba de control para identificar una expresión como acto: una expresión que permita ser introducida por dicho verbo será un acto".

8 Por supuesto, como se puede comprobar en el análisis de cualquier conversación coloquial, no es este el único nivel en que funcionan los conectores: también pueden funcionar con análogo papel demarcativo en el nivel dialogico $\mathrm{y}$, en general, a nivel interactivo.
} 
(5)

A: la culpa de todo la tuvo Juan

$\mathrm{B}$ : bueno/ pero él lo hizo sin mala intención

Un grupo especial de modalizadores es el de los llamados reguladores fáticos, donde se integran unidades como yo qué sé, no sé, ¿sabes?, ¿no?, ¿eh? etc. En general, este tipo de elementos no llegan a constituir Actos independientes:

(6)

8 E4: \#bien yo qué sé- yo por ejemplo $\uparrow$ / no sée// a mí me parece muy bien lo que hace cada uno $\downarrow \#$ \#que yo no estoy de acuerdo $\uparrow$ no quiere decir que yo le critique ni que no $/ /{ }^{\circ}(\text { ¿entiendes? })^{\circ} \#$

Funcionan más bien como modalizadores que atenúan ocasionalmente la respuesta, a modo de paréntesis del Acto, como en (7)

(7)

19 G6: \#hombre liberal/ lo que pasa es que no sé pues/ tú a lo mejor entiendes por liberal puess\#/// \#(hay) gente que entiende $\rightarrow$ pues un viva la virgen ¿no?\# \#o sea// que pasan de todo\# que- que- que

De cualquier forma, habitualmente no debemos pensar en estas unidades (¿sabes?, ¿no?, ¿eh?, no sé, etc.) como preguntas que el oyente vaya a responder o como aseveraciones más o menos categóricas. Suelen tener escaso o nulo valor apelativo (o aseverativo), aunque a veces lo posean'. Si su función es la de meros reguladores o elementos de refuerzo deberemos interpretarlos como Subactos constituyentes de un constituto superior, el Acto.

Por lo demás, algunas de las llamadas conjunciones de coordinación plantean problemas particulares para la segmentación de Actos, ya que tienden (según su valor semántico) hacia un constituyente u otro.

P.e. en el caso de $y$, una cuestión fundamental es determinar si lo que une son dos segmentos equifuncionales, tales comodos pronombres relativos en (8):

21 G7: \#eso tampoco es\#/ \#una persona liberal $\uparrow$ para mí es una persona que tienee/ unos principios ¿no? y quee/ oye/ intenta cumplirlos $\downarrow$ a rajatabla ¿no?/// simplemente\#/ y bueno yyy

en cuyo caso hablaremos de un solo Acto y no de dos; en este contexto lo que une $y$ forma parte de un mismo contenido proposicional, dos Subactos. Del mismo modo, si de lo que se trata es de la relación asindética de complementos, como en (9):

(9)

21 G7: \#eso tampoco es\#/ \#una persona liberal $\uparrow$ para mí es una persona que tienee/ unos principios ¿no? y quee/ oye/ intenta cumplirlos $\downarrow$ a rajatabla ¿no?/// simplemente\#/ y bueno yyy

9 En este último caso habremos de considerarlos como Actos; p.e. si hubiera una respuesta efectiva por parte del oyente. 
seguiremos hablando de un único Acto (incluyendo el adverbio "simplemente"), ya que un complemento de un núcleo verbal no puede, en principio, formar un Acto independiente.

En cambio, si $y$ enlaza contenidos proposicionales distintos, hablaremos de dos Actos, como en $(10)$ :

12 E6: \#yo tengo unos principios\# y \#para mí hay valores fundamentales\#

\section{2. Índices lingüísticos suprasegmentales demarcativos de Actos}

La prosodia es en ocasiones el único recurso demarcativo con que cuenta el analista para proceder a la segmentación del discurso en Actos. En este sentido un criterio prosódico demarcativo evidente, al menos en apariencia, es la pausa. Otra cuestión es si realmente esa marca delimita unidades estructurales coherentes, lo cual puede resultar más discutible.

Al respecto, la opinión de los estudiosos parece oscilar en torno a dos grandes posturas, la de aquellos que defienden la correlación entre la presencia de una pausa (silencio) y de una estructura sintáctica determinada (Bally, 1941; Gardiner, 1951; Alarcos, 1974: 107,111), y la de aquellos que minimizan la correlación entre la pausa y la organización sintáctica (Bloomfield, 1933: 221; Gili Gaya, 1951: 110 y 1978: 49).

Desde el punto de vista estrictamente sintáctico, suele afirmarse que el empleo de la pausa está en razón inversa del uso que hace la lengua de los elementos conjuntivos (así, la ausencia de elementos gramaticales conjuntivos determinaría, hipotéticamente, un aumento de la duración pausal). De este modo, según su duración la pausa sería capaz de establecer jerarquías de unidades sintácticas (Esser, 1987; Cruttenden, 1990: 36-39; Cazanove, 1988: 225; Clark-Yallop, 1990: 282). Parece razonable, pues, aceptar que el funcionamiento de la pausa está relacionado con requerimientos verbales diferentes: aumenta su frecuencia con la complejidad semántica y decrece su presencia a medida que aumenta el grado de planificación discursiva.

Discrepan de las opiniones anteriores quienes prefieren reservar el funcionamiento regular de las pausas para estilos discursivos más formales o planificados. Tal es el caso de Gili Gaya (1951: 110), para quien el carácter simple o compuesto de una oración (esto es, su rango jerárquico) poco tiene que ver con la realización o no de pausa: la división en grupos fónicos o el carácter unitario de una secuencia de habla dependen simplemente de la voluntad del hablante.

Lo cierto es que la pausa no siempre determina la existencia de frontera entre unidades, esto es, entre Actos. Puede ser simplemente un mecanismo de planificación o retardatario en la conversación, como ocurre en (11) con la pausa entre "menos" y "es", o en (12) con las pausas entre "tal" y "precisamente" y "valores" y "muy":

17 G5: \#a ti te da lo mismo ¿no?\#/\#pues más o menos/ es eso lo- lo que quiere decir más o menos la palabra liberal\#

22 E11: \#es que- es que ee yo para mî $\uparrow$ el hecho de ser conservadores y taal / precisamente radica en sus principios\#/ y \#para mi $\uparrow$ hay unas- unos valores // muy fundamentales que a lo mejor para otra persona no lo son ino?/// (3") no sé\# 
La contextualización del uso de la pausa es, en cualquier caso, requisito indispensable para la delimitación adecuada de los Actos en la conversación; véase así la diversidad de funciones y valores de las pausas en $6 \mathrm{E} 3$ de (13), donde no parece evidenciarse una correlación clara entre duración de pausa y jerarquía de unidad delimitada:

(13)

4 E2: \#síi\#/ \#yo conozco gentee\#/ \#parezco muy liberal pero// la verdad es que soy muy conservadora\#

5 L3: \#mujer/ en todo no $\downarrow^{\circ}$ (tía $)^{\circ} \#$

6 E3: \#yo si\# / \#liberaal- soy conservadora enn-/ pues en lo que interesa como to'l mundo\#// \#pero vamos no soy nada liberal $\downarrow \#$ \#lo contrario\# /// \#lo que pasa $\neq$ es que yo respeto mucho lo que dice la gente $\downarrow \#$ \#a mí- cada uno que haga lo que quiera yy\#

Al margen de la presencia o no de pausa, la posesión de una curva melódica completa puede constituirse como señal inequívoca de la existencia de un Acto: tal es el caso de las formas melódicas interrogativa o exclamativa, formas lingüísticas que constituyen, pues, marcas de la presencia de Actos, y no sólo por los signos de interrogación o exclamación que aíslan la construcción, sino por el valor modal que tales signos vienen a expresar (pregunta, rechazo, etc.).

Consecuentemente, la importancia del componente suprasegmental como instrumento de segmentación obliga a que, en el caso de ciertos segmentos, sea absolutamente imprescindible prestar atención al funcionamiento específico de su curva melódica. Así, expresiones como ;mujer!, ;hombre!, jvamos!, etc. pueden constituirse en Actos independientes siempre que sus respectivos contornos melódicos se articulen aisladamente, esto es, cuando no se hallen integrados en una curva principal. Ello explica que en (14) se manifiesten comportamientos prosódicos demarcativos bien diferentes a los de (15) con relación al segmento "hombre":

19 G6: \#hombre liberal/ lo que pasa es que no sé pues/ tú a lo mejor entiendes por liberal puess\#/// \#(hay) gente que entiende $\rightarrow$ pues un viva la virgen ino?\# \#o sea// que pasan de todo\# que- que- que

18 E9: \#hombre!\# \#yo- o sea yo por liberal $\uparrow$ no entiendo esto\#

Es conveniente, en tales casos, tener muy en cuenta las magnitudes de los respectivos parámetros prosódicos (acento, duración, frecuencia fundamental, etc.) ${ }^{10}$. En suma, ni la pausa que establece límites entonativos, ni las fronteras entonativas por sí mismas constituyen un límite absoluto. Sólo armonizando criterios externos e internos podemos considerar la pausa como frontera.. La pausa adquiere valor en la medida en que, cuando

10 Obsérvese por otra parte que las formas melódicas básicas pueden desarrollar innumerables valores modales expresivos o determinados contextualmente, lo que representa una muestra más de la responsabilidad notable de los suprasegmentos en la adecuada delimitación e interpretación de los Actos (Hidalgo, 1998, 2002a y 2002b). 
aparece, se yuxtapone a máximos o mínimos prosódicos que traducen una o varias funciones comunicativas ${ }^{11}$.

Al margen de los rasgos prosódicos que expresan la existencia de una curva melódica completa (y por tanto de un Acto aislable), a lo largo del discurso oral espontáneo son frecuentes las rupturas de la construcción en curso, lo que puede ocasionar serias dudas en cuanto a la segmentación adecuada del fragmento en cuestión. Es conveniente, pues, aclarar cuándo, ante tales circunstancias, un determinado fragmento constituye Acto y cuándo no.

Tal es el caso de cierto tipo de Actos cuya estructura apocopada está condicionada por la intencionalidad del hablante. En tales circunstancias el carácter "aparentemente" incompleto del Acto obedece a un propósito organizativo de naturaleza pragmática, es decir, a una "estrategia" preconcebida. Tales Actos reciben la denominación de Actos estratégicos, y una de sus manifestaciones más habituales son los denominados Actos suspendidos.

Efectivamente, los Actos suspendidos configuran estructuras gramaticalmente inacabadas, pero comunicativamente completas. Poseen una entonación final marcada (tonema de ascenso o suspensión). Constituyen un uso convencional y estratégico por parte del emisor, en consecuencia, sí configuran Acto. La construcción suspendida es intencional, no se suple contextual, sino pragmáticamente; véase así 16 E8 en (16):

12 E6: \#yo tengo unos principios\# y \#para mí hay valores fundamentales\#§

$13 \mathrm{G} 3$ : $\S \#$ \#so\#/ \#y tú

los sigues ¿no?\#

14 E7: $\quad \S \#$ sï\#

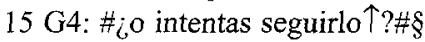

$16 \mathrm{E} 8$ :

$\S \#$ \#ï\#/ \#pero que otra persona no los sigaa\#

Con el Acto suspendido el hablante pretende que el oyente infiera aquello que pretende comunicarle realmente. Se presupone que, en este caso, el hablante sabe que su oyente tiene el conocimiento suficiente para inferir su intención. El oyente deberá hacer, pues, cierto sobreesfuerzo cognitivo, pero no hay ningún elemento externo que pueda verse como causa del corte en lo comunicado: el oyente reconstruye a través de su memoria enciclopédica. La construcción suspendida se basta a sí misma; lingüísticamente, pues, es un Acto completo.

En otros casos la existencia de reinicios, autocorrecciones, vacilaciones de habla, etc. no representa necesariamente un obstáculo para que el segmento afectado pueda constituirse en Acto; hablamos en este caso de Actos truncados. Así, si bien por lo general los reinicios son reelaboraciones que plantean problemas de planificación: si tras ese cambio existe un fin, una intención (una estrategia), debemos pensar que son efectivamente Actos, como es el caso de $2 \mathrm{E} 1$ en (17):

11 Por lo demás, como se ha comentado anteriormente, la pausa no constituye un fenómeno limitado al silencio; hay otros fenómenos relacionados con la no continuidad enunciativa propios del habla espontánea. Deben tenerse en cuenta, incluso, factores más complejos como la propia intencionalidad y disponibilidad conceptual del hablante (aspectos psicolingüísticos). Además, el fenómeno pausal no se comporta en la conversación exclusivamente como mecanismo demarcativo-segmentador de Actos en el Nivel Monológico, sino que desarrolla funciones más complejas de índole interactiva (interrupciones, solapamientos, finales de turno, finales de tópico, etc.), relacionadas con el Nivel Dialógico. 
(17)

1 L1: \#yo creo que- no sé $\downarrow$ que tienes actos muy- muy liberales [en relación a]\# $2 \mathrm{E} 1$ : \#[no soy nada-]\# \#no son

liberales\#

Efectivamente, el interlocutor puede llegar a procesar el contexto merced a este tipo de truncamiento, ya que poseen la capacidad de aislabilidad contextual. Frente a los Actos suspendidos, aquí no hay inferencia alguna que hacer: el oyente puede completar el acto a través del contexto, pero no interpreta más allá de lo que dice el hablante, ni el hablante pretende que lo haga. Exigen menor esfuerzo cognitivo al oyente que los Actos suspendidos, ya que en los Actos truncados el contexto ayuda a suplir lo que no aparece, ausencia que obedece a una estrategia intencional por parte del hablante; p.e., en ocasiones puede haber detrás de este mecanismo un propósito atenuador, como en 19G6 de (18), donde se pasa de la segunda persona del singular a la expresión impersonal:

18 E9: \#¡hombre!\# \#yo- o sea yo por liberal $\uparrow$ no entiendo esto\#§ 19 G6:

$\$$ \#hombre liberal/ lo que pasa es que no sé pues/ tú a lo mejor entiendes por liberal puess\#/// \#(hay) gente que entiende $\rightarrow$ pues un viva la virgen ¿no?\# \#o sea// que pasan de todo\# que- que- que-

\section{Definición y reconocimiento de subactos}

\subsection{Estatuto jerárquico del subacto. Actos simples y actos complejos}

De lo anteriormente expuesto se deduce que el segmento mínimo identificable con valor estructural en el nivel monológico es el Subacto. Se trata de un elemento jerárquicamente inferior al Acto por lo que, inicialmente, puede considerarse como constituyente inmediato del Acto.

Debe entenderse así que un Acto puede, bien constituirse en sí mismo como estructura acabada (en cuyo caso el Acto se llena con un solo segmento informativo) o bien estar integrado por dos o más Subactos (dos o más segmentos informativos). Hablaremos así de dos tipos de Actos desde el punto de vista de su constitución interna, Actos Simples y Actos Complejos.

Los Actos Simples configuran en sí mismos un Acto completo, sin Subactos reconocibles como constituyentes inmediatos. Los Actos Complejos, en cambio, están constituidos por más de un Subacto (dos o más), en cuyo caso sí se puede hablar de constituyentes inmediatos del Acto, ya que en su interior aparecen dos o más unidades informativas mínimas reconocibles o segmentables.

En ocasiones, sin embargo, en el curso de su emisión el hablante escinde ciertos segmentos cuyo estatuto jerárquico plantea alguna confusiones para su segmentación. Nos referimos a los fenómenos de dislocación (DSL) o topicalización (TOP) (véase Padilla, 2001a y $2001 \mathrm{~b})^{12}$. El caso de las DSL es bastante claro, ya que su vinculación sintáctica con el resto

12 En general, de acuerdo con Padilla (2002) consideramos como DSL, sea a la izquierda o a la derecha, toda estructura que presenta los rasgos [+clítico referencial], [+se mueve un $\mathrm{OD} / \mathrm{OI}$ ], [-se mueve un elemento distinto de OD/OI). En la topicalización en cambio se cumplen los rasgos [-clítico referencial], [-se mueve un OD/Ol], [+se mueve un elemento distinto de Objeto]. 
del Acto (o construcción sintáctica en curso) impide de forma inmediata que la podamos considerar como Acto en sí mismo:

(19)

A: síi/ echa/ este tronco lo mandamos a tomar por culo

Ahora bien, la presencia habitual de una breve pausa (cuantificada como pausa de duración inferior a medio segundo en el corpus publicado por el grupo Val.Es.Co.), como marca de separación entre una TOP y el segmento del que se desgaja, podría hacernos pensar en un Acto diferente. De cualquier forma, la escasa duración de esta pausa no parece criterio suficiente para establecer la existencia de dos Actos diferentes:

B: es un chorizo de mucho cuidado/ ese

La TOP será considerada, pues, como parte integrante del Acto. Ahora bien, si la duración de la pausa es más amplia y, sobre todo, significativa estructuralmente, cabe la posibilidad de considerar dos Actos diferentes, en cuyo caso se descartaría la existencia de TOP.

Por lo general, pues, los elementos topicalizados o dislocados (a la derecha o a la izquierda) no deben considerarse nunca como Actos, a lo sumo como Subactos (véase al respecto Padilla, 2002).

Se entiende, en definitiva, que el Subacto es una unidad informativa, para ser más exactos, un segmento informativo mínimo; su valor puede ser el de justificación, condición, causa, TOP, DSL, etc., de modo que los Subactos pueden llegar a mantener entre sí relaciones jerárquicas de algún modo equiparables con las de la sintaxis (unos son, informativamente hablando, más importantes que otros).

\subsection{Delimitación de Subactos. Articulación prosódica del Acto en Subactos}

Aspecto fundamental para la delimitación del Subacto es considerarlo desde el punto de vista de su articulación prosódica; efectivamente, paso previo a la identificación de su valor informativo es su delimitación por medios prosódicos, ya que todo Subacto configura siempre un grupo de entonación ${ }^{13}$. Los mecanismos prosódicos deben ser considerados consecuentemente como señales demarcativas de primera importancia, esto es, como índices inequívocos para la posible segmentación de un Acto en dos o más Subactos, es decir, de la posible escisión estructural del Acto en dos o más partes delimitadas prosódicamente (entonativamente, para ser más exactos), constitutivas cada una de ellas de un grupo de entonación.

Ello capacita al grupo de entonación para constituirse por sí mismo como segmento mínimo de habla reconocible en la conversación coloquial, lo que le confiere el carácter de instrumento demarcativo operativo, y aplicable sin restricciones en el proceso de demar-

\footnotetext{
13 Nuestra visión de grupo de entonación coincide con la de Quilis y otros (1993: 56-57), esto es, "la porción de discurso comprendida entre dos pausas, entre pausa e infiexión del fundamental, entre inflexión del fundamental y pausa, o entre dos inflexiones del fundamental que configura una unidad sintáctica más o menos larga o compleja (sintagma, cláusula, oración)".
} 
cación de Subactos. Sin embargo, no todo grupo de entonación representa necesariamente un Subacto; más exacto sería decir que la forma lingüística más habitual de representación del Subacto es el grupo de entonación ${ }^{14}$. Por otro lado, como también ha quedado señalado previamente, el Acto puede estar constituido por uno o más grupos de entonación; pues bien, es precisamente en este último caso, cuando el Acto integra dos o más grupos de entonación, cuando entran en juego las relaciones estructurales de índole prosódica entre tales grupos de entonación ( $\mathrm{o}$, en su caso, entre tales Subactos).

En este sentido, la altura tonal de la sílaba final del grupo entonativo (esto es, la inflexión melódica o tonema) constituye un criterio estructural de primera importancia, ya que aporta información sobre la naturaleza de la relación de dicho segmento con el grupo entonativo que le sigue inmediatamente. Así, una entonación final baja (descendente) suele manifestar la autonomía entre ambos grupos entonativos; en el siguiente ejemplo $1 \mathrm{~B}$ y $2 \mathrm{~B}$ son grupos entonativos autónomos y, además, Actos independientes. De ește modo, ante la secuencia de habla "si ya- si por ahí hay cartelitos $\downarrow$ lo que pasa es que $\rightarrow / / /$ está a medio colocar aún $\downarrow$ ", cabría proponer la siguiente estructuración:

1B: si ya- si por ahí hay cartelitos $\downarrow$

2B: lo que pasa es que $\rightarrow / / /$ está a medio colocar aún $\downarrow$

(A. Hidalgo, 1996: 474; 85-86)

Cada unidad entonativa con final en tono bajo (descendente) por relación a su punto de ataque mantiene, pues, una relación de yuxtaposición con la unidad entonativa vecina. Hablaríamos en este caso de dos Actos distintos, a lo que contribuiría decisivamente el criterio estructural prosódico. A su vez, una entonación alta al final de un primer constituyente (I), manifiesta una relación de encajonamiento o dependencia con el siguiente (II), y así sucesivamente; en este caso, se puede decir que (I) determina entonativamente (II). El segmento que acaba con tono alto $(\uparrow)$ se interpreta como señal por relación al segmento siguiente, que constituye el término señalado, de acuerdo con la propuesta del Grupo de la Sorbona ${ }^{15}$ :

C: (I) porque ellos $\uparrow$ (I) (II) se repartieron las tres mesas y me dejaron para que yo estuviera allí tranquila $\downarrow$ (II) (A Hidalgo, 1996: 485; 612-613)

De este modo, la estructura resultante del Acto tiene carácter recursivo ya que gran número de secuencias discursivas configuran Actos articulados (constituidos por dos o más

14 Es posible hallar grupos de entonación que no son ni Actos ni Subactos, sino simplemente segmentos fragmentarios de habla; esto ocurre, como hemos señalado previamente en los reinicios, autocorrecciones, etc. que configuran sin duda grupos de entonación, pero al margen de la estructura discursiva: son meras vacilaciones. Véase en cambio el caso de las construcciones suspendidas donde un grupo de entonación melódicamente inconcluso (abierto en anticadencia o suspensión), representa por sí mismo un Acto perfectamente inferible para el oyente.

15 El Grupo de La Sorbona está constituido por un número importante de investigadores interesados en la estructura del "enunciado oral espontáneo" (cfr. al respecto Morel y Rialland, 1992; Danon-Boileau y otros, 1991; Danon-Boileau y otros, 1992; Morel, 1992a y 1992b; Morel, 1993a, 1993b y 1993c). 
grupos de entonación) que se encajonan entonativamente los unos a los otros para construir el discurso ora ${ }^{16}$.

\section{Hacia una clasificación funcional del subacto}

Una vez asumida la función demarcativa de los elementos prosódicos y determinada su relevancia para llegar a reconocer posibles Subactos en el interior de un Acto, cabe formular microestructuras discursivas capaces de articular un Acto y de integrarlo como unidad comunicativa, de acuerdo con la estrecha relación que tales microestructuras contraen con los mecanismos de presentación informativa ${ }^{17}$. Lo importante, pues, es asumir, como se indicaba anteriormente, la consideración del Subacto como unidad informativa al tiempo que unidad prosódica (grupo de entonación). Así, a partir del análisis efectuado por Val.Es.Co. cabe dilucidar la existencia de ciertas estructuras habituales en el seno del Acto, esté articulado o no por Subactos ${ }^{18}$.

\subsection{Articulación interna del Acto}

\subsubsection{Acto constituido por un único Subacto (Acto Simple)}

Si se da esta situación el Acto constituye un grupo de entonación único, una sola forma melódica completa. Puede tratarse de una forma melódica neutra (acabada), como es el caso de los Actos identificables en las intervenciones de $\mathrm{E}$ y $\mathrm{G}$ en (23):

E: \#yo tengo unos principios\# y \#para mí hay valores fundamentales\# $\S>\underline{2 \text { Actos }}$

$\mathrm{G}$ :

$\S$ \#吕\#I \#y tú los

E:

sigues $i n o ? \#>\underline{2 \text { Actos }}$

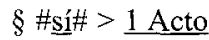

o de una forma melódica "aparentemente" inacabada (suspendida, truncada, etc. ${ }^{19}$, como es el caso del segundo Acto en la segunda intervención de G en (24):

E: \#yo tengo unos principios\# y \#para mí hay valores fundamentales\#§

G: $\S$ \#eso\#/ \#y tú los sigues $\measuredangle$ no?\#§

\footnotetext{
16 Las reglas de jerarquización entonativa presentan, como se puede ver, ventajas claras en el análisis del habla espontánea, donde no siempre hay marcas sintácticas, léxicas o morfológicas capaces de explicar las relaciones entre las distintas partes de un Acto; en estos casos la organización interna parece indiicarse suficientemente sólo mediante lazos entonativos.

17 En la consideración de estos fenómenos seguimos los trabajos de Danon-Boileau y otros (199l), DanonBoileau y otros (1992) y Morel y Rialland (1992).

18 Ha de advertirse, en cualquier caso, que las construcciones que vamos a comentar constituyen parte de un estudio en proceso, por lo que el modelo de integración propuesto deberá ser matizado e implementado en el curso de sucesivas investigaciones futuras.

19 Sobre la diversidad estructural de estas formas melódicas "aparentemente" inacabadas y constitutivas de Actos completos, véanse Briz y otros (2003).
} 
E:

G: \# o intentas seguirlos $\uparrow ? \# \S$

$\S$ \#Si\#

E: $\S$ \#si\#/ \#pero que

otra persona no los sigaa \#

\subsubsection{Acto constituido por dos o más Subactos (Acto Complejo)}

En este caso atendemos fundamentalmente al valor informativo de los Subactos, y por tanto, a la capacidad de los recursos prosódicos (fundamentalmente de la entonación) para articular informativamente el discurso. Se observan así distintos tipos de Subactos de acuerdo con su valor informativo; inicialmente hemos propuesto dos clases esenciales, los Subactos Sustantivos, y los Subactos Adyacentes.

\subsubsection{Subactos Sustantivos}

Son segmentos constitutivos de Acto que poseen efectivamente contenido informativo (lo que podríamos denominar "sustancia" informativa). Son, pues, unidades llenas que disponen de contenido "proposicional" (algo que se predica de algo o de alguien), de modo que aportan la información "esencial" del Acto.

Tal como se entienden los Subactos Sustantivos, es posible diferenciar dos tipos específicos según su papel estructural en la organización interna del Acto. Hablaremos así de Subactos Sustantivos Directores (SSD) y Subactos Sustantivos Subordinados (SSS). E1 SSD resulta potencialmente aislable y equivale (semántica e informativamente) a la esencia misma del Acto, esto es, a su conjunto. Por su parte, el SSS se halla supeditado funcionalmente al SSD, en la idea de que suele aportar algún tipo de dependencia, ya sea semántica, ya sea pragmática, respecto de dicho SSD. Véase así el ejemplo (25):

\section{(25)}

A: ¿vienes al cine?

B: no (SSD), porque tengo prisa (SSS)

En cualquier caso, no puede haber más que un SSD en un mismo Acto, pues en caso contrario ya no estaríamos hablando de un solo Acto, sino de dos (o más). No obstante, ello no impide que un Acto determinado pueda ser descompuesto en varios Subactos Sustantivos.

Esta distinción funcional puede materializarse de diversas formas, p.e., la segmentación producida entre el tema y el rema en el interior de un Acto, o entre una oración principal y una oración subordina en el período complejo, o incluso entre una primera oración coordinada copulativa y una segunda coordinada copulativa en el período compuesto. Así, p.e. el tema, grupo de entonación substantivo (es decir, con sustancia semántico-informativa) que suele ocupar la posición de segmento señal, presenta por su parte una entonación ascendente (anticadencia o semianticadencia), como en la primera intervención de B en (26):

B: sí $\downarrow$ eso $\uparrow$ (tema) =]

A: $\quad$ [mira este $\downarrow$ Albacete $]$

B: = eso sí que lo sé (A. Hidalgo, 1996: 494; 53-55) 
En todos estos casos hay un único Acto, pero segmentado en dos Subactos. Hablamos entonces de dos segmentos potenciales del Acto: el Subacto Señal o primera parte del Acto y el Subacto Señalado o segunda parte del Acto. La relación ulterior que pueda establecerse entre estos elementos podrá ser diversa, pero lo importante es que ambos, Señal y Señalado, sean entendidos como grupos de entonación informativamente positivos, por eso se consideran como grupos de entonación (y además como Subactos) Sustantivos: ambos representan aporte informativo efectivo.

\subsubsection{Subactos Adyacentes}

La segmentación posible de un Acto no se agota con los Subactos Sustantivos (SSD o SSS), ya que también existen otras estructuras constitutivas por debajo del Acto. Nos referimos a los Subactos Adyacentes.

Los Subactos Adyacentes no aportan información esencial para el Acto en que se integran, sino más bien información al margen. Son, en este sentido, elementos "extraproposicionales", en la medida en que no forman parte de la estructura proposicional. Por lo demás, de acuerdo con la matización específica que aporten a la proposición es posible distinguir varios tipos fundamentales. En este sentido, algunos de los llamados marcadores discursivos, entre los que figurarían formas como pero, porque, sin embargo, además, oye, vamos, entonces, etc., desarrollan una diversidad funcional en cuanto a su comportamiento demarcativo que conviene comentar.

\subsection{Subactos Adyacentes Textuales}

Pueden expresar p.e. lazos con un texto o emisión anterior: desde el punto de vista comunicativo, marcan al interlocutor que se le ha escuchado, y proporcionan el tiempo necesario para planificar lo que se va a decir; desde el punto de vista enunciativo, sitúan lo que se va a decir frente a lo ya dicho. Algunos marcadores, pues, deben ser entendidos como una clase específica de marcas reguladoras, capaces de organizar y distribuir el flujo del habla, que no representan por sí mismas aporte semántico. Constituyen por tanto un tipo particular de Subactos Adyacentes, que podemos denominar Textuales ${ }^{20}$.

Por otro lado, un marcador puede desarrollar papeles funcionales diferentes según su realización prosódica específica ( \pm prominente) $)^{21}$. Así, un conector alto puede p.e. marcar el límite de una unidad mínima, esto es, el final de un Acto y el comienzo del siguiente, representando asi un papel anafórico y/o argumentativo. Obsérvese al respecto el valor argumentativo, introductor de un nuevo Acto, presente en el marcador entonces en (27) ${ }^{22}$.

\footnotetext{
20 Más exactamente, de acuerdo con Chafe (1993), se trataría de un subtipo de grupos de entonación reguladores textuales, que pueden llegar a ocupar un contorno entonativo propio y que expresan vínculos de naturaleza diversa entre lo que antecede y lo que sigue, ya sea en el interior de una misma intervención, ya sea entre intervenciones correspondientes a hablantes diferentes.

21 En el ámbito hispánico, si bien desde una perspectiva más auditiva que acústica, A. Briz (1993: 150-161) ha reconocido igualmente la participación decisiva de la prosodia en la delimitación de la función sintáctico-pragmática de algunos "conectores". Sobre tal diversidad cfr. también A. Briz y A. Hidalgo (1998).

22 En los ejemplos (27) y (28) entre paréntesis aparece indicado el valor que alcanza en ese punto la Frecuencia Fundamental (F0), magnitud físico-acústica que se mide en hercios $(\mathrm{Hz})$.
} 
(27)

B: ya $\downarrow$ pero si me dices eso ya $\uparrow$ estamos tergiversando los resultados $\downarrow$

A: no $\downarrow$ porque yo realmente $\uparrow$ no me doy cuenta de que has apretado el botón que has apretado $^{\wedge}<$ entonces $\uparrow(260 \mathrm{hz})$ estoy hablando con toda la [naturalidad=]

B:

A: $=$ del mundo $\downarrow$ te lo prometo $\downarrow>$ (A. Hidalgo, 1996: 473; 6-10)

Efectivamente, comprobamos aquí que una realización prosódica con prominencia elevada $\left(260 \mathrm{~Hz}\right.$ de $\mathrm{F}_{0}$ ) sobre entonces favorece la autonomía y aislabilidad del segmento "entonces $\uparrow$ estoy hablando con toda la naturalidad del mundo $\downarrow$ te lo prometo $\downarrow$ " con respecto del segmento precedente "no $\downarrow$ porque yo realmente $\uparrow$ no me doy cuenta de que has apretado el botón que has apretado^" ".

Un conector bajo (es decir, un marcador sin prominencia tonal) suele funcionar en cambio como enlace sintáctico puramente local. No marcará, pues, el comienzo de un nuevo Acto, sino en su caso el comienzo de un Subacto (Subacto Señalado), tal como ocurre con el marcador pero (emitido a una $\mathrm{F}_{0}$ de $200,2 \mathrm{~Hz}$ ) en (28), donde $\mathrm{C}$ continúa un mismo Acto en dos intervenciones diferentes:

(29)

C: hay truuucos (( )) hay muchos trucos ahí $\downarrow$ que yo todavía no los tengoo [(( )) controlaos $\uparrow=]$

B: [apretar bo-

toncitos]

$\mathrm{C}:=$ pero $\rightarrow(200,2 \mathrm{hz}) / /$ sí $\downarrow$ pero hay otros $\uparrow$ que no $\rightarrow$ tengo todavía muy claro $\downarrow$ (A. Hidalgo, 1996: 488; 725-729)

Esquemáticamente la estructura del Acto articulado por C en (28) sería:

hay truuucos hay muchos trucos ahí $\downarrow$ que yo todavía no los tengoo controlaos $\uparrow>$ SUBACTO SEÑAL

pero $\rightarrow / /$ si $\downarrow$ pero hay otros $\uparrow$ que no $\rightarrow$ tengo todavía muy claro $\downarrow>$ SUBACTO SEÑALADO

\section{(Donde ACTO = SUBACTO SEÑAL + SUBACTO SEÑALADO)}

\subsection{Subactos Adyacentes Interpersonales}

A los tipos anteriores se añade un grupo que representa la interacción entre hablante y oyente y que podríamos considerar como grupos reguladores interpersonales, esto es, Subactos Adyacentes Interpersonales. Nos referimos con ello a segmentos de diversa naturaleza:

- unidades como mm, si, oh, ya, etc., siempre que funcionen en la conversación como muestras de atención del oyente hacia su interlocutor, como en la segunda intervención de L en (29): 
(29)

L: \#yo también pienso que la- la religión es importante $\uparrow$ / peroo tú te la- la puedes entender de una forma o de otra/l/ ¿sabes?\#§

E:

$\S$ \#él es/ tranqui tranqui\#

$\mathrm{L}: \mathrm{mm}$

E: o sea $\rightarrow$

L: ((en determinados momentos))

- interrogaciones fáticas del tipo ¿sabes?, ¿no? ¿eh?, etc., cuando actúen como recursos apelativos del hablante hacia el oyente, como en (30):

(30)

L: \#yo también pienso que la- la religión es importante $\uparrow /$ peroo tú te la- la puedes entender de una forma o de otra/// ¿sabes?\#

\subsection{Subactos Adyacentes Modalizadores}

Estos Subactos Adyacentes introducen algún tipo de matización específica sobre el Subacto Sustantivo al que se adhieren (atenuación, actitud del hablante ante lo dicho, etc.); funcionan, pues, como modalizadores del discurso, por lo que los hemos denominado Subactos Adyacentes Modalizadores (SAM). Ejemplos de este grupo son algunas expresio-

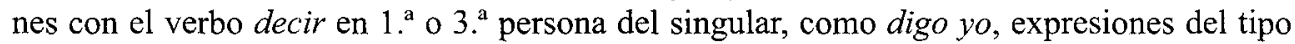
no sé, yo qué sé, etc.

Obsérvese que en algún caso un SAM puede encabezar el Acto de modo que podría ser catalogados como "preacto" (lo que, entre otras cosas, podría hacernos pensar en un tipo específico de SAM):

(31)

E: (...) \#yo qué sé/ no se trata de ser/ extremista o a rajatabla y de aquí ya $\uparrow$ \#

En otras ocasiones estos SAM expresan explícitamente el punto de vista del emisor sobre su discurso ${ }^{23}$ :

B: pero no dec- no decía nada

A: nada $\downarrow$ nada $\downarrow$ y yo $\rightarrow$ pero iqué aburrimiento! de verdad $\downarrow$ y (O) eres una persona francamente aburrida $\downarrow$ y además $\downarrow$ sé quién eres ¿sabes?

(A. Hidalgo, 1996: 517; 139-141)

\section{Conclusiones}

Planteadas las anteriores posibilidades estructurales, podemos afirmar con certeza que en la conversación coloquial resulta viable la identificación de una serie de unidades mínimas,

23 Obsérvese que en tales casos el SAM parece manifestar características prosódicas especificas (p.e. sucle realizarse con una entonación descendente y prominencia tonal elevada). Morfosintácticamente puede estar representado por diversas categorías: un adverbio o expresión adverbial de modalidad, un pronombre personal, una fórmula verbal, un grupo conjuntivo complejo, etc. 
los Actos, que se integran en el marco de las Intervenciones y que a su vez se hallan constituidos por segmentos inferiores de naturaleza prosódico-informativa, los Subactos.

De acuerdo con la estructura inherente de cada Acto podemos establecer una distinción entre Actos simples (constituidos por un único segmento informativo o Subacto Sustantivo) y Actos complejos (constituidos por dos o más segmentos informativos o Subactos que pueden mantener entre sí diversas relaciones específicas, con la condición sine qua non de que entre tales segmentos informativos se localice un Subacto Sustantivo como mínimo).

Por lo demás, en el seno de un Acto complejo cabe identificar uno o varios segmentos "subordinados" al Subacto Sustantivo, a los que hemos llamado Subactos Adyacentes, por su carácter informativamente "subsidiario". Para la delimitación de tales Subactos Adyacentes es fundamental tener en cuenta la participación de los elementos suprasegmentales o entonativos. Efectivamente, el análisis entonativo permite aclarar las relaciones entre los distintos elementos que configuran el Acto complejo, fundamentalmente las existentes entre un Subacto Adyacente Textual, un Subacto Sustantivo Director y un Subacto Sustantivo Subordinado virtuales, esto es, entre los potenciales subactos integrantes del Acto. A tales subactos se añaden otras posibles subestructuras reguladoras de la progresión discursiva (Subactos Adyacentes), según la complejidad interna del Acto constitutivo ${ }^{24}$, entre las cuales destacan por su frecuencia los Subactos Adyacentes Interpersonales y los Subactos Adyacentes Modalizadores.

\section{Referencias bibliográficas}

Alarcos, E. (1974): Fonologia Española. Madrid, Gredos.

Bally, Ch. (1941): "Intonation et syntaxe", Cahiers Ferdinand de Saussure, 1, págs. 33-42.

Bloomfield, L. (1933): Lenguaje. Lima, Universidad Nacional Mayor de San Marcos. (Citado por la edición de 1964).

Briz, A. (1993): "Los conectores pragmáticos en la conversación coloquial (I): su papel argumentativo", Contextos XI/21-22, págs. 145-188.

Briz, A. (2000a): “Las unidades de la conversación”. En Briz, A. y Grupo Val.Es.Co. (2000): ¿Cómo se comenta un texto coloquial? Barcelona. Ariel. págs. 51-80

Briz, A. (2000b): "Turno y alternancia de turno en la conversación", Revista Argentina de Lingïística 16 , págs. 3-26.

Briz, A. (en prensa): "Las unidades de la conversación: el acto". En Homenaje a J.J. Bustos Tovar.

Briz, A. (coord.) (1995): La conversación coloquial. Materiales para su estudio. Anejo XVI de Cuadernos de Filologia. Valencia, Universitat de València.

Briz, A. (coord.) (2002): Corpus de conversaciones coloquiales. Anejo I de la revista Oralia. Madrid. Arco Libros.

Briz, A. e Hidalgo, A. (1998): "Conectores y estructura de la conversación". En Martín Zorraquino, M. A. y Montolío, E. (eds.): Marcadores del discurso. Teoría y análisis. Madrid. Arco Libros. págs. 121-142.

Briz, A. y Grupo Val.Es.Co. (en prensa): "Acerca de la estructura conversacional del español coloquial", en Actas del XXIII Congreso Internacional de Lingüistica y Filología Románicas. 2001. Salamanca.

Briz, A. y otros (2003): "Un sistema de unidades para el estudio del lenguaje coloquial", en Oralia, 6. Cazanove, M. T. (1988): "Phénomenes de pause", Revue de Phonétique Appliquée, 87/89, págs. 223246.

Clark, J. y C. Yallop (1990): An Introduction to Phonetics and Phonology. London, Basil Blackwell. 
Chafe, W. (1993): "Prosodic anf Functional Units of Language". En Edwards, J. A. y Lampert, M. D. (eds.): Transcription and coding in discourse research. New Jersey. Lawrence Erlbaum Associates. págs. 34-39.

Cruttenden, A. (1990): Entonación. Barcelona, Teide.

Danon-Boileau, L. y otros (1991): "Intégration discursive et intégration syntaxique", Langages, 104, págs. 111-128.

Danon-Boileau, L. y otros (1992): Intonation et structure de l'énoncé oral. Colloque Gréco-Dialogue Homme-machine, Dourdan, (Monografia de Langages, $\mathrm{n}^{\circ} 104$ ).

Esser, J. (1987): "Functions of Intonation". En AA.VV.: Functionalism in Linguistics. Amsterdam, Benjamins. págs. 381-393.

Gardiner, A. (1951-1989): Langage et acte du langage. Aux sources de la pragmatique. The theory of speech and Language. Lille. Presses universitaires de Lille.

Gili Gaya, S. (1951). "Fonología del periodo asindético". En Estudios dedicados a Menéndez Pidal. Madrid, Consejo Superior de Investigaciones Científicas. págs. 55-67.

Gili Gaya, S. (1978): Elementos de fonética general. Madrid, Gredos. ( $1^{\text {a }}$ Ed. 1966).

Gutiérrez, S. (1984): ¿Es necesario el concepto de oración?", RSEL, 14/2, págs. 245-270.

Hidalgo, A. (1996): Entonación y conversación coloquial. Sobre el funcionamiento demarcativo-integrador de los rasgos suprasegmentales. Tesis Doctoral. Universitat de València.

Hidalgo, A. (1997): Entonación coloquial. Función Demarcativa y Unidades de Habla. Valencia. Anejo XXI de la revista Cuadernos de Filología. Universitat de València.

Hidalgo, A. (1998): "Expresividad y función pragmática de la entonación en la conversación coloquial. Algunos usos frecuentes", Oralia, 1, págs. 69-92.

Hidalgo, A. (2002a): Comentario fónico de textos coloquiales. Madrid. Arco Libros.

Hidalgo, A. (2002b): "Modalidad oracional y entonación. Notas sobre el funcionamiento pragmático de los rasgos suprasegmentales en la conversación", Moenia, 7, págs. 271-292.

Morel, M. A. (1992a): "Intonation et thématisation", L'information grammaticale, 54. Monografía de Langage, $\mathrm{n}^{\circ} 104$.

Morel, M. A. (1992b): "L'opposition thème-rhème dans la structuration des dialogues oraux", French Language Studies, 2, págs. 85-98.

Morel, M. A. (1993a): "Intonation interrogative et structure repère/repéré". Colloque CERLICO, Nantes.

Morel, M. A. (1993b): "Valeur énonciative des variations de hauteur mélodique (points hauts el points bas)". Workshop Pragmatics, discourse and 'énonciation', Manchester.

Morel, M. A. (1993c): "L'enoncé oral complexe. Les relatives en QUI", Travaux de linguistique du CERLICO, 6, págs. 1-21.

Morel, M. A. y A. Rialland (1992): "Emboîtement, autonomies, ruptures dans l'intonation française", en Travaux Linguistiques du CERLICO, 5, págs. 221-243.

Narbona, A. (1986): "Problemas de sintaxis coloquial andaluza", en RSEL 16/2, págs. 229-275.

Padilla, X. A. (2001a): "Orden de palabras y español coloquial: estrategias sintácticas, semánticas e informativas". En Actas del I Congreso Internacional de Análisis del Discurso (Lengua, Discurso, Texto). Vol. I. Madrid. Visor. págs. 823-836.

Padilla, X. A. (2001b): El orden de palabras en el español coloquial. Tesis doctoral. Inédita. Universitat de València.

Padilla, X. A. (2002): "Las unidades monológicas (intervención, acto y subacto) y el orden de palabras: una guía para su análisis en el discurso hablado", comunicación presentada en el Simposio Análisis del Discurso (Lengua, Cultura, Valores). Pamplona, 26-28 de nov. de 2002.

Pons, S. (2001): "Las unidades de la conversación", conferencia ofrecida en las Jornadas de Pragmática. Universitat de València, 6-8 de noviembre de 2001.

Quilis, A. y otros (1993): "El grupo fónico y el grupo de entonación en español hablado", Revista de Filología Española, 73, págs. 55-64. 